\title{
Which Drug to Discontinue 3 Months After Combination Therapy of Tadalafil plus Tamsulosin for Men with Lower Urinary Tract Symptom and Erectile Dysfunction? Results of a Prospective Observational Trial
}

\author{
Arcangelo Sebastianelli $^{a}$, Pietro Spatafora ${ }^{a}$, Jacopo Frizzi $^{a}$, Omar Saleh $^{a}$, Cosimo De Nunzio ${ }^{b}$, \\ Andrea Tubaro ${ }^{b}$, Linda Vignozzi $^{c}$, Mario Maggi ${ }^{c}$, Sergio Serni ${ }^{a}$, Kevin T. McVary ${ }^{d}$, \\ Steven A. Kaplan ${ }^{e}$, Stavros Gravas ${ }^{f}$, Christopher Chapple ${ }^{g}$, Mauro Gacci $^{a, *}$ \\ ${ }^{a}$ Department of Minimally Invasive and Robotic Urologic Surgery and Kidney Transplantation, University of Florence, Florence, Italy; ${ }^{\mathrm{b}}$ Department of \\ Urology, Sant'Andrea Hospital, University “La Sapienza”, Rome, Italy; ${ }^{\mathrm{c}}$ Department of Clinical Physiopathology, University of Florence, Florence, Italy; ${ }^{\mathrm{d}}$ Center \\ for Male Health, Department of Urology, Stritch School of Medicine, Loyola University Medical Center, Maywood, IL, USA; ${ }^{\mathrm{e} D e p a r t m e n t ~ o f ~ U r o l o g y, ~ I c a h n ~}$ \\ School of Medicine at Mount Sinai, New York City, NY, USA; ${ }^{\mathrm{f}}$ Department of Urology, University of Thessaly, Larissa, Greece; ${ }^{\mathrm{g}}$ Department of Urology, Sheffield \\ Teaching Hospitals NHS Trust, Sheffield, UK
}

\section{Article info}

Article history:

Accepted September 24, 2019

Associate Editor:

Dr. Malte Rieken

Keywords:

Benign Prostatic Obstruction Benign Prostatic Hyperplasia Erectile Dysfunction lower urinary tract symptoms combination therapy tadalafil tamsulosin

\begin{abstract}
Background: Safety and efficacy of tamsulosin and tadalafil for men with benign prostatic enlargement (BPE) and/or erectile dysfunction (ED) are defined. However, there are only a few pilot studies on combination therapy with these drugs for men with lower urinary tract symptom (LUTS)/BPE and ED. Moreover, preliminary reports are limited to $12 \mathrm{wk}$, without any information about subsequent therapies.

Objective: To evaluate the impact of discontinuation of tamsulosin versus tadalafil 12 wk after combination therapy.

Design, setting, and participants: Fifty consecutive patients with moderate-to-severe LUTS (International Prostate Symptom Score [IPSS] > 7) and mild-to-severe ED (International Index of Erectile Function-5 [IIEF-5] < 22) were treated with combination therapy (tamsulosin $0.4 \mathrm{mg} / \mathrm{d}$ plus tadalafil $5 \mathrm{mg} / \mathrm{d}$ ) for $12 \mathrm{wk}$. After $12 \mathrm{wk}, 25$ patients discontinued tamsulosin (Group TAD), while 25 patients discontinued tadalafil (Group TAM).

Outcome measurements and statistical analysis: Efficacy variables were IPSS (total, voiding, storage) and IIEF-5. Paired samples $t$ test and analysis of variance were used. Results and limitations: Groups TAD and TAM presented similar features (age, BMI, metabolic profile) including symptoms scores at baseline. Similar and significant improvements in IPSS (total, voiding, and storage) and IIEF-5 were recorded in both groups after 12 wk of combination therapy (all $p<0.001$ ). Total IPSS was similar between the two groups at the end of the trial. However, we found between-group significant differences from baseline to $24 \mathrm{wk}$ and from 12 to $24 \mathrm{wk}$ in storage-IPSS (Group TAD: -3.32 vs Group TAM: $-1.24, p=0.002$; Group TAD: +0.24 vs Group
\end{abstract}

\footnotetext{
* Corresponding author at: Department of Minimally Invasive and Robotic Urologic Surgery and Kidney Transplantation, University of Florence, Largo Brambilla 3, Florence, 50134, Italy. Tel. +39055275 8011, Fax: +390552758014.

E-mail addresses: maurogacci@yahoo.it, maurogacci@aou-careggi.toscana.it (M. Gacci).
}

https://doi.org/10.1016/j.euf.2019.09.015

2405-4569/@ 2019 European Association of Urology. Published by Elsevier B.V. All rights reserved. 
TAM: $+1.20, p=0.040$, respectively) and in IIEF-5 (Group TAD: +4.64 vs Group TAM: +0.16, $p<0.001$; Group TAD: -1.64 vs Group TAM: $-4.40, p=0.003)$. No significant treatment-related adverse event was recorded in both groups.

Conclusions: After 12 wk of combination therapy, monotherapy with tadalafil for further $12 \mathrm{wk}$ allows to preserve the improvement of storage IPSS and IIEF-5, in addition to total IPSS.

Patient summary: In this report we evaluated the discontinuation of tamsulosin or tadalafil after $12 \mathrm{wk}$ of combination therapy. We found that tadalafil monotherapy, for a further $12 \mathrm{wk}$, aids in retaining the improvement of storage symptoms and erectile function.

(c) 2019 European Association of Urology. Published by Elsevier B.V. All rights reserved.

\section{Introduction}

Lower urinary tract symptoms (LUTSs) and erectile dysfunction (ED) are significant health concerns that will increase in the next years in relation to the aging of the population. Both conditions have a significant impact on the overall male quality of life (QoL). LUTS, including voiding, storage, and postmicturition symptoms, secondary to benign prostatic hyperplasia (BPH) is frequently reported in middleaged or older men. They range from mild to severe and could have a major impact on patients' QoL $[1,2]$.

ED, defined as a persistent inability to achieve and maintain an erection sufficient for satisfactory sexual performance, is generally considered the result of para-aging factors and its prevalence has increased from 5\% for men in their forties to $15 \%$ for those aged $70 \mathrm{yr}$ [3].

Several large-scale epidemiological studies in different population have demonstrated a relationship between LUTS and ED in aging men that is independent of the effects of age or other comorbidities; particularly, men with severe LUTS presented an incidence of ED about three times higher than those without LUTS $[1,4]$.

Today, although the molecular and pathological pathways potentially linking LUTS and ED are not clearly defined, several possible biological mechanisms including an altered nitric oxide/cyclic guanosine monophosphate pathway, an altered RhoA/Rho kinase signaling, systemic inflammation, pelvic ischemia, autonomic adrenergic hyperactivity, or overactivity have been investigated [5-8].

LUTS/BPH medical treatment may also have a significant impact on erectile function (EF). Several randomized controlled trials demonstrated that alpha-blockers (ABs) and 5alpha reductase inhibitors, the most commonly prescribed drugs for managing patients with LUTS/BPH, can be associated with sexual adverse effects (AEs) such as decreased or loss of libido, ED, and ejaculatory dysfunction [9-11].

Phosphodiesterase type 5 inhibitors (PDE5-Is), the firstline therapy for ED, and particularly tadalafil $5 \mathrm{mg}$ once daily, have been approved and considered an effective and well-tolerated treatment for LUTS [10].

Although the exact mechanism of action of PDE5-Is remains unclear, several preclinical and clinical trials have shown that they can reduce the smooth muscle tone of detrusor, prostate, and urethra together with an increased blood perfusion and oxygenation in the lower urinary tract; besides, its possible effect on chronic inflammation in the prostate and bladder has been proposed [12-15].

Safety and efficacy of PDE5-Is are well defined and combination therapy of PDE5-Is and ABs, particularly tamsulosin, the only AB approved by the Food and Drug Administration in combination with tadalafil, has shown to have an additive favorable effect on International Prostate Symptom Score (IPSS), International Index of Erectile Function-5 (IIEF-5) score, and maximum flow rate $\left(\mathrm{Q}_{\max }\right)$ compared with monotherapy alone [16]. So far, the recent possibility to manage both LUTS and ED by PDE5-i alone or in combination with tamsulosin may open up new management strategies, considering that treatment of one condition may have an impact on the other. However, although a combination therapy is considered a promising and tailored approach seeking a balance between efficacy and tolerability in patients with LUTS, there are no data on when to move from a single-drug management to a combination approach and what are the possible changes in efficacy by switching from one treatment modality to another [17].

The aim of this observational study is to evaluate the impact of discontinuation of tamsulosin $(0.4 \mathrm{mg})$ or tadalafil ( $5 \mathrm{mg}$ ) after $12 \mathrm{wk}$ of combination therapy in patients with LUTS and ED.

\section{Material and methods}

\subsection{Design and participants}

A prospective observational trial was designed and performed. Across $12 \mathrm{mo}, 50$ consecutive patients with ED and LUTS suggestive of benign prostatic obstruction (BPO) were enrolled.

Inclusion criteria were age $>40-80 \mathrm{yr}$, mild to severe ED (IIEF-5 $<22$ ), moderate to severe LUTS (IPSS $>7$ ), and $\mathrm{Q}_{\max }>5 \mathrm{ml} / \mathrm{s}$ (obtained from a uroflowmetry assessment). Exclusion criteria were hypersensitivity to tadalafil or tamsulosin, prostatic cancer or suspected with prostate-specific-antigen $>4.0 \mathrm{ng} / \mathrm{ml}$, bladder lithiasis, previous prostatic surgery, urinary tract infection, neurogenic bladder, finasteride or dutasteride use within 6 mo, and a clinical history of urethral and/or proven bladder neck obstruction.

The study was performed in accordance with applicable laws and regulations, good clinical practices, and ethical principles as described in the Declaration of Helsinki. 
Institutional Review Boards for each site approved the study (Ethics Committee Approval OSS.15.031). All men provided written informed consent before initiating any trial procedure or therapy.

The assessment of patients included age, body mass index, waist circumference, blood pressure, clinical laboratory parameters, digital rectal examination, IPSS [18], IIEF-5 score [19], uroflowmetry, and postvoid residue (PVR) volume (evaluated with abdominal ultrasound immediately after voiding for uroflowmetry). The IPSS storage and voiding subscores, nocturia question, and IPSS-QoL index were also assessed.

After a 4-wk wash-out period from previous therapies for BPO and a 2-wk run-in period with tamsulosin $0.4 \mathrm{mg}$, patients were treated in two urologic centers with combination therapy of tamsulosin $0.4 \mathrm{mg} / \mathrm{d}$ and tadalafil $5 \mathrm{mg} / \mathrm{d}$ for $12 \mathrm{wk}$.

After $12 \mathrm{wk}$, the tamsulosin therapy was discontinued in one center, leaving 25 patients with tadalafil $5 \mathrm{mg} / \mathrm{d}$ alone (Group TAD), whereas tadalafil was discontinued in the other center, leaving 25 patients with tamsulosin $0.4 \mathrm{mg} / \mathrm{d}$ only (Group TAM; Fig. 1).

Patients were evaluated at screening time, after $12 \mathrm{wk}$ of combination therapy, and after 12 wk of monotherapy.

Patients were instructed to self-administer the study drugs at the same time every day before sleep, without restrictions of food intake or timing of sexual activity. Patients were considered compliant if at least 90\% of the drug amount was taken. Safety was assessed by evaluating patient-reported AEs, orthostatic vital signs, PVR, uroflowmetry, and clinical laboratory parameters. Patients with incomplete data sets were excluded from statistical analysis.

The variables considered for measuring the efficacy of treatment were the changes in IPSS and its subscores (total, voiding, and storage), the modification of IIEF-5 score, and the improving of $\mathrm{Q}_{\max }$. All follow-up visits, including uroflowmetry, were performed in the morning (from 8:00 am to $12: 00 \mathrm{pm})$.

\subsection{Statistical analysis}

Differences between combination therapy and the two groups switched to monotherapy were calculated by an unpaired sample $t$ test at baseline, $12 \mathrm{wk}$, and $24 \mathrm{wk}$, respectively. Mean changes between baseline and $12 \mathrm{wk}$ and those between 12 and 24 wk were assessed by a paired sample $t$ test for each treatment group. Between-group differences in change from baseline to $12 \mathrm{wk}$ and from 12 to 24 wk were measured by one-way analysis of variance (ANOVA). All statistical analyses were performed with SPSS (SPSS Inc., Chicago, IL, USA). A $p$ value of 0.05 or less was considered statistically significant.

\section{Results}

A total of 50 patients were treated with combination therapy of tamsulosin $0.4 \mathrm{mg} / \mathrm{d}$ plus tadalafil $5 \mathrm{mg} / \mathrm{d}$ for 12 wk [20]. Subsequently, 25 patients continued tadalafil 5 $\mathrm{mg} / \mathrm{d}$ (Group TAD) and 25 continued tamsulosin $0.4 \mathrm{mg} / \mathrm{d}$ (Group TAM) only, for a further 12 wk. A Consolidated Standards of Reporting Trials (CONSORT) flowchart is shown in Fig. 1. All participants completed the study. Compliance with dosing requirements was over $90 \%$ in both groups. Both treatment groups were well balanced, without significant differences in metabolic features, age, and BPO-related characteristics (Table 1). The overall improvement from baseline to the first checkpoint after 12 wk of combination therapy (tadalafil plus tamsulosin) was statistically significant for all the items evaluated in

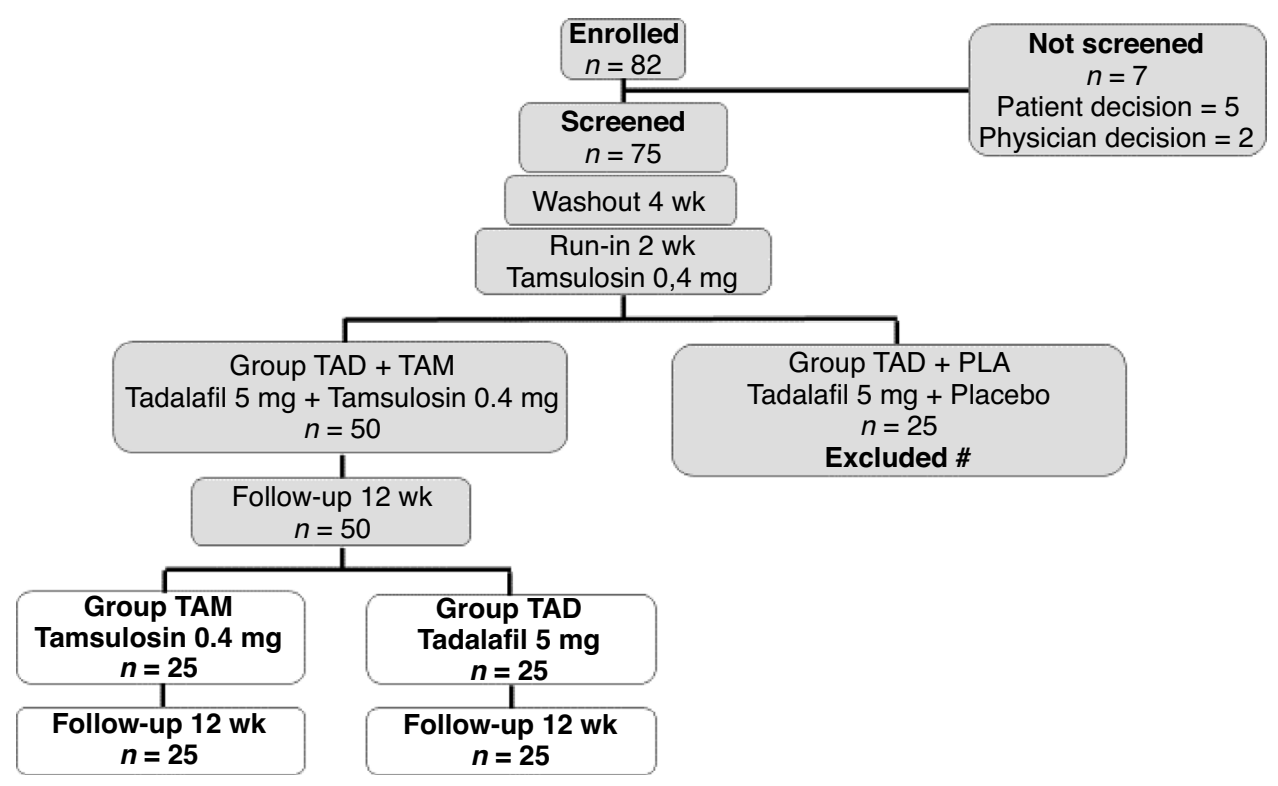

Fig. 1 - Disposition of patients. Patient Consolidated Standards of Reporting Trials (CONSORT) diagram.

PLA = placebo.

a Patients who did not perform combination therapy of tadalafil plus tamsulosin for 12 weeks did not continue the study. 
Table 1 - Patients' baseline characteristics.

\begin{tabular}{|c|c|c|c|c|c|}
\hline & Group TAD $(n=25)$ & & Group TAM $(n=25)$ & & \\
\hline & Mean \pm standard deviation & Minimum-Maximum & Mean \pm standard deviation & Minimum-Maximum & $p$ value \\
\hline Age (yr) & $64.4 \pm 8.3$ & $48-77$ & $67.0 \pm 7.8$ & $47-78$ & 0.314 \\
\hline Weight (kg) & $77.3 \pm 8.2$ & $67-85$ & $75.9 \pm 9.5$ & $66-83$ & 0.306 \\
\hline Body mass index $\left(\mathrm{kg} / \mathrm{m}^{2}\right)$ & $27.2 \pm 5.3$ & $24-32$ & $25.9 \pm 5.7$ & $25-32$ & 0.187 \\
\hline $\begin{array}{l}\text { Abdominal obesity: } \\
\text { waist circumference }(\mathrm{cm})\end{array}$ & $107.8 \pm 6.1$ & $93-133$ & $104.3 \pm 4.5$ & $78-128$ & 0.142 \\
\hline Triglycerides (mg/dl) & $164.4 \pm 6.4$ & $78-268$ & $138.7 \pm 6.8$ & $81-203$ & 0.128 \\
\hline HDL cholesterol (mg/dl) & $48.7 \pm 3.2$ & $28-68$ & $49.2 \pm 2.6$ & $31-62$ & 0.275 \\
\hline Glycemia (mg/dl) & $123.2 \pm 4.5$ & $79-224$ & $107.7 \pm 5.7$ & $79-215$ & 0.124 \\
\hline IPSS base & $18.1 \pm 5.8$ & $8-29$ & $19.6 \pm 6.1$ & $11-32$ & 0.369 \\
\hline IPSS voiding base & $8.3 \pm 4.3$ & $1-16$ & $8.9 \pm 3.4$ & $5-20$ & 0.537 \\
\hline IPSS storage base & $8.8 \pm 3.2$ & $0-14$ & $7.9 \pm 3.2$ & $2-13$ & 0.354 \\
\hline IPSS QoL base & $3.9 \pm 0.9$ & $3-6$ & $3.8 \pm 1.2$ & $2-6$ & 0.686 \\
\hline IIEF-5 base & $12.3 \pm 4.0$ & $6-21$ & $11.8 \pm 3.2$ & $6-18$ & 0.587 \\
\hline $\mathrm{Q}_{\max }$ base $(\mathrm{ml} / \mathrm{s})$ & $14.0 \pm 3.8$ & $8.4-23.0$ & $11.8 \pm 4.1$ & $3.4-19.7$ & 0.55 \\
\hline
\end{tabular}

our population: total, voiding, and storage IPSS; IIEF-5; and $\mathrm{Q}_{\max }($ all $p<0.001$ ). Between-group ANOVA, comparing Group TAD only versus Group TAM only, did not show significant differences for total IPSS $(p=0.233)$, storage IPSS $(p=0.235)$, voiding IPSS $(p=0.02)$; IIEF-5 $(p=0.412)$; and $\mathrm{Q}_{\max }(p=0.908$; Table 2). As shown in Table 3, after 12 wk of monotherapy, a statistically significant difference was found between the two groups for mean IPSS storage score (Group TAD: +0.24 vs Group TAM: $+1.2 ; p=0.040$; Fig. 2A), mean IIEF-5 score (Group TAD: -1.64 pts vs Group TAM: $-4.4 ; p=0.003$; Fig. $2 \mathrm{~B}$ ), and mean $\mathrm{Q}_{\max }$ (Group TAD: $-2.41 \mathrm{ml} / \mathrm{s}$ vs Group TAM: $-0.25 \mathrm{ml} / \mathrm{s} ; p=0.001$; data not shown). Conversely, total IPSS and voiding IPSS were similar after the switch from combination to monotherapy (Fig. 3A and $3 \mathrm{~B}$ ). The proportion of patients reporting at least one treatment-emergent $\mathrm{AE}$ (TEAE) was similar between groups (TAD: 16\% vs TAM: 20\%; Table 4). TEAEs were mild to moderate in severity, with the most common being headache and back pain. There were no clinically significant changes in laboratory measurements or vital signs. No urinary retention was reported. None of the patients discontinued therapy because of a TEAE.

\section{Discussion}

Currently, there are four PDE5-Is approved for ED treatment which have been investigated for the treatment of LUTS with or without ED in randomized double-blind clinical trials. In a recent meta-analysis, combination of PDE5-is and ABs improved the IIEF-5 score $(+3.6 ; p<0.001)$, IPSS $(-1.8 ; p=0.05)$, and $\mathrm{Q}_{\max }(+1.5 \mathrm{ml} ; p<0.001)$ when compared with ABs alone [21].

The authors concluded that, although the cost-effectiveness analysis of this treatment is needed, PDE5-Is are effective and well tolerated either alone or in combination with ABs, particularly in young patients with severe LUTS/BPH. Moreover, a recent prospective urodynamic study evaluating the impact of daily tadalafil $5 \mathrm{mg}$ on storage and voiding function in male BPH patients showed that mean bladder outlet obstruction index significantly decreased from 59.5 at baseline to 45.7 at $3 \mathrm{mo}(p=0.001)$, and to 42.9 at $12 \mathrm{mo}(p<0.001)$ [22].

Thus, tadalafil once daily, alone or in combination with tamsulosin, is increasingly prescribed in clinical practice as an effective therapy for LUTS/BPE and concomitant ED $[23,24]$.

Table 2 - Change from baseline to 12 wk for Group TAD and Group TAM.

\begin{tabular}{llll}
\hline & Group TAD $(n=25)$ & Group TAM $(n=25)$ & $p$ value* \\
\hline Mean IPSS 12 wk & 10.4 & 12.6 \\
Delta3 M (baseline-12 wk) & -7.64 & -6.96 & 5.72 \\
Mean IPSS voiding 12 wk & 4.52 & -3.24 \\
Delta3 M (baseline-12 wk) & -3.76 & 5.48 \\
Mean IPSS storage 12 wk & 5.2 & -2.44 \\
Delta3 M (baseline-12 wk) & -3.56 & 2.04 \\
Mean IPSS QoL 12 wk & 2.24 & -1.8 \\
Delta3 M (baseline-12 wk) & -1.72 & 16.88 \\
Mean IIEF-5 12 wk & 18.6 & +5.12 & 0.233 \\
Delta3 M (baseline-12 wk) & +6.28 & 13.97 \\
Mean Qmax 12 wk & 18.28 & +2.21 \\
Delta3 M (baseline-12 wk) & +4.27 & 0.235 \\
\hline IPSS = International Prostate Symptom Score; IIEF-5 $=$ International Index of Erectile Function-5; Qmax $=$ maximum urinary flow rate; QoL $=$ quality of life. \\
Levels of significance are calculated with paired sample $t$ test $(p \leq 0.05)$. The $p$ value indicates the level of significance as per analysis of variance. \\
\hline
\end{tabular}


Table 3 - Change from 12 wk to 24 wk for TAD and TAM groups.

\begin{tabular}{|c|c|c|c|}
\hline & Group TAD $(n=25)$ & Group TAM $(n=25)$ & $p$ value ${ }^{*}$ \\
\hline Mean IPSS 24 wk & 12.48 & 14.32 & 0.386 \\
\hline Delta6 M (12-24 wk) & +2.08 & +1.72 & \\
\hline Mean IPSS voiding $24 \mathrm{wk}$ & 6.2 & 6.36 & 0.456 \\
\hline Delta6 M (12-24 wk) & +1.68 & +0.64 & \\
\hline Mean IPSS storage $24 \mathrm{wk}$ & 5.44 & 6.68 & 0.040 \\
\hline Delta6 M (12-24 wk) & +0.24 & +1.2 & \\
\hline Mean IPSS QoL 24 wk & 2.88 & 2.56 & 0.628 \\
\hline Delta6 M (12-24 wk) & +0.64 & +0.52 & \\
\hline Mean IIEF-5 24 wk & 16.96 & 12.48 & 0.003 \\
\hline Delta6 M (12-24 wk) & -1.64 & -4.4 & \\
\hline Mean $Q_{\max } 24$ wk & 15.87 & 13.72 & 0.001 \\
\hline Delta6 M (12-24 wk) & -2.41 & -0.25 & \\
\hline
\end{tabular}

(A)

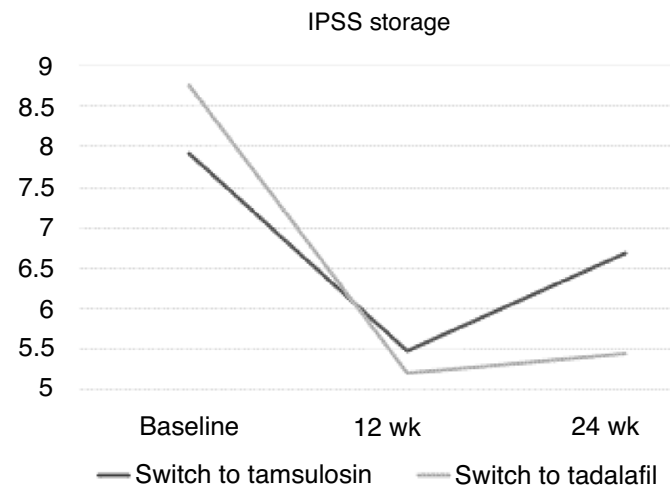

(B)

IIEF-5

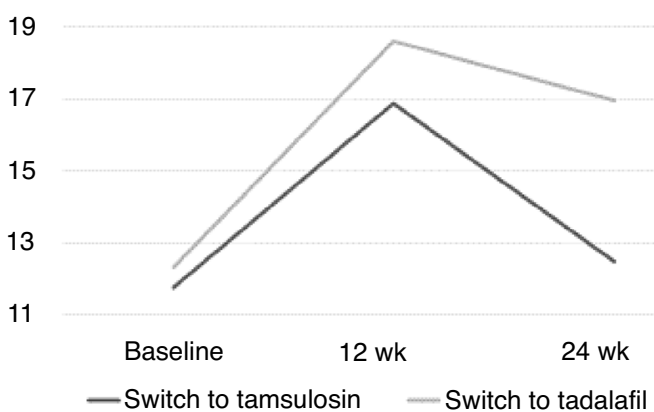

Fig. 2 - Change from baseline to 24 wk for Groups TAD and TAM.

Group TAD = discontinued tamsulosin; Group TAM, discontinued tadalafil; IIEF-5 = International Index of Erectile Function-5; IPSS = International Prostate Symptom Score; $Q_{\max }=$ maximum urinary flow rate.

(A)

IPSS total

21

19

17

17
15

13

11

9

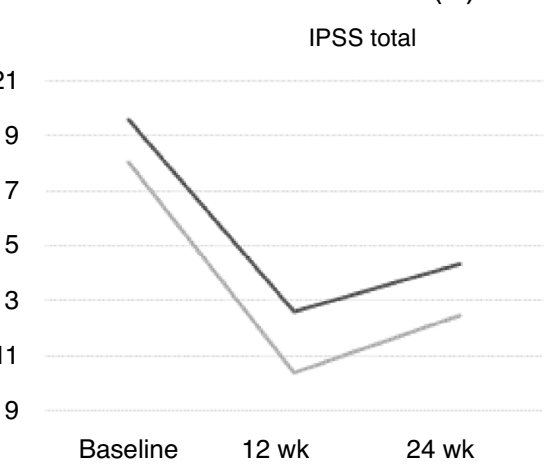


Table 4 - Summary of adverse events reported during the treatment period ( $24 \mathrm{wk}$ ).

\begin{tabular}{lll}
\hline & $\begin{array}{l}\text { Group TAD } \\
(n=25), n(\%)\end{array}$ & $\begin{array}{l}\text { Group TAM } \\
(n=25), n(\%)\end{array}$ \\
\hline Any TEAEs & $4(16)$ & $5(20)$ \\
Serious AEs & $0(0.0)$ & $0(0.0)$ \\
\hline Intensity & & \\
Mild & $3(12)$ & $3(12)$ \\
Moderate & $1(4)$ & $2(8)$ \\
Severe & $0(0.0)$ & $0(0.0)$ \\
Headache & $2(8)$ & $2(8)$ \\
Nasopharyngitis & $0(0.0)$ & $1(4)$ \\
Back pain & $1(4)$ & $1(4)$ \\
Dizziness & $0(0.0)$ & $0(0.0)$ \\
Dyspepsia & $1(4)$ & $0(0.0)$ \\
\hline Ejaculatory dysfunction & $0(0.0)$ & $1(4)$ \\
\hline AEs = adverse events; TEAEs $=$ treatment-emergent adverse events. \\
\hline
\end{tabular}

placebo, although their results were not clinically relevant, because a mean change less than 3 points in IPSS was recorded.

In our study, patients in the TAD group experienced a statistically significant but not clinically relevant ( $<1$-point) change in storage IPSS when compared with patients in the TAM group. However, in both groups we observed a statistically and clinically significant improvement from baseline. Our results confirmed the long-term effect of PDE5-i treatment on LUTS, particularly storage LUTS and that this effect is maintained for at least $12 \mathrm{wk}$ when treatment is suspended.

The urodynamic findings of Matsukawa et al [22] support our results. Indeed, in 49 patients who had detrusor overactivity during cystometry at the baseline assessment, uninhibited detrusor contractions disappeared in 15 (30.6\%) after $3 \mathrm{mo}(p=0.02)$, and in $22(44.9 \%)$ after $12 \mathrm{mo}(p<0.001)$ of treatment with tadalafil.

Moreover, tadalafil proved to be an alternative add-on drug for patients with persistent storage LUTS refractory to $\alpha_{1}$-adrenoceptor antagonists, even when compared with solifenacin, thus confirming the valuable effect of tadalafil on storage LUTS [26].

Patients in the TAM group retained the improvement of $\mathrm{Q}_{\max }$ achieved with combination therapy (13.97 at $12 \mathrm{wk}$ vs 13.72 at $24 \mathrm{wk}$ ). Conversely, a decrease of $\mathrm{Q}_{\max }$ was observed in the TAD group (18.28 at $12 \mathrm{wk}$ vs 15.87 at $24 \mathrm{wk}$ ); however, an improvement of $\mathrm{Q}_{\max }$ from baseline was also observed in the TAD group ( $14.0 \mathrm{vs} 15.87 \mathrm{ml} / \mathrm{s}$ ).

In contrast with our results, the first systematic review by Laydner et al [27] on the use of PDE5-Is for BPE-related LUTS reported that PDE5-Is improved IPSS and IIEF-5 but not $Q_{\max }$.

Moreover, in another meta-analysis, PDE5-Is were more effective in association with ABs, improving IPSS (mean difference -1.8 ), IIEF-5 score (mean difference +3.6 ), and $\mathrm{Q}_{\max }$ (mean difference $+1.5 \mathrm{ml} / \mathrm{s}$ ), when compared with PDE5-Is alone, which improved IPSS and IIEF scores but not $\mathrm{Q}_{\max }[16]$.
However, Dong et al [25] concluded that even after pooling four doses $(2.5,5,10$, and $20 \mathrm{mg})$, tadalafil failed to produce a significant outcome in $Q_{\max }$, whereas $5 \mathrm{mg} / \mathrm{d}$ tadalafil therapy significantly improved $\mathrm{Q}_{\max }$ (mean difference $=+0.63 \mathrm{ml} / \mathrm{s}, p=0.04$ ).

As expected, only patients in the TAD group retained the IIEF-5 improvement when compared with the TAM group (16.96 vs $12.48 ; p=0.003$ ). Interestingly, the impact of tadalafil on IIEF-5 was more pronounced, even if not clinically significant, in combination with tamsulosin $(+6.28$ vs +4.64$)$, suggesting a possible synergism between these drugs. Indeed, a previous prospective randomized study demonstrated that EF improved with tamsulosin $(+39.28 \%[p<0.05])$, tadalafil $(+45.96 \%$ $[p<0.05])$, and tamsulosin and tadalafil combination $(+60.23 \%[p<0.05])$, with a better improvement seen in combination therapy compared with single agent alone. However, in line with our findings, the impact of combination treatment on EF was significantly greater than tamsulosin alone $(p<0.05)$ but not when compared with tadalafil $(p=0.125)[28]$.

In our study, we described for the first time urinary and sexual outcomes achieved within $12 \mathrm{wk}$ of tamsulosin or tadalafil monotherapy after a previous $12 \mathrm{wk}$ of combination therapy, to achieve a tailored therapy based on patients' predominant symptoms and to reduce drugs' intake.

As previously reported for the combination therapy of tamsulosin and dutasteride, the withdrawal of tamsulosin after 24 wk of combination therapy had no significant effect on urinary outcomes. Indeed, LUTS relief was maintained in the majority of patients after tamsulosin was removed from combination, and the authors concluded that only patients with severe symptoms may benefit from longer-term combination therapy [29].

Even if combination therapy with tamsulosin and tadalafil achieved better urinary and sexual outcomes, after a further $12 \mathrm{wk}$ of monotherapy, both drugs were able to preserve a significant recovery of urinary function from baseline. Nevertheless, only patients treated with tadalafil showed a satisfying sexual function and a better storage IPSS at the end of the trial.

Daily doses of tadalafil $5 \mathrm{mg}$ and tamsulosin $0.4 \mathrm{mg}$ were well tolerated. No patients discontinued the study because of AEs. Headache was the main AE, with a higher incidence in the TAM group (8\%) as compared with the TAD group (4\%), suggesting that the use of tamsulosin can increase the risk of this AE.

Strengths of the study are the prospective nature of the trial and data collection together with the homogenous population. However, this study may be restricted by some limitations. Because this study was observational, it could be prone to biases. Moreover, prostate size was not considered as inclusion/exclusion criteria or as a potential determinant on the efficacy of therapy. Long-term follow-up is also needed to better evaluate the role of a switching approach versus a continuous treatment in terms of patients' compliance in term of symptoms, EF, urinary flow management and costs. 


\section{Conclusion}

For men with LUTS and ED, combined treatment with tamsulosin and tadalafil is well tolerated and effective. After 3 mo of combination therapy and consequent discontinuation of tamsulosin, monotherapy with tadalafil was able to further preserve the improvement of LUTS and EF although with a slight decrease of $\mathrm{Q}_{\max }$. The possibility to switch from a combination approach to a single-treatment strategy seems a feasible option to improve patients' compliance and to better tailor LUTS treatment.

\author{
Author contributions: Mauro Gacci had full access to all the data in the \\ study and takes responsibility for the integrity of the data and the \\ accuracy of the data analysis. \\ Study concept and design: Gacci. \\ Acquisition of data: Frizzi. \\ Analysis and interpretation of data: Gacci. \\ Drafting of the manuscript: Sebastianelli, Spatafora. \\ Critical revision of the manuscript for important intellectual content: De \\ Nunzio, Vignozzi. \\ Statistical analysis: Sebastianelli. \\ Obtaining funding: None. \\ Administrative, technical, or material support: Saleh. \\ Supervision: Serni, Tubaro, Maggi. \\ Other: visualization: McVary, Kaplan, Gravas, Chapple.
}

Financial disclosures: Mauro Gacci certifies that all conflicts of interest, including specific financial interests and relationships and affiliations relevant to the subject matter or materials discussed in the manuscript (eg, employment/affiliation, grants or funding, consultancies, honoraria, stock ownership or options, expert testimony, royalties, or patents filed, received, or pending), are the following: None.

\section{Funding/Support and role of the sponsor: None.}

\section{References}

[1] Gacci M, Eardley I, Giuliano F, et al. Critical analysis of the relationship between sexual dysfunctions and lower urinary tract symptoms due to benign prostatic hyperplasia. Eur Urol 2011;60:809-25.

[2] De Nunzio C, Roehrborn CG, Andersson KE, et al. Erectile dysfunction and lower urinary tract symptoms. Eur Urol Focus 2017;3:352-63.

[3] NIH Consensus Conference. Impotence. NIH consensus development panel on impotence. JAMA 1993;270:83-90.

[4] Shiri R, Hakkinen JT, Hakama M, et al. Effect of lower urinary tract symptoms on the incidence of erectile dysfunction. J Urol 2005;174:205-9.

[5] Anderson KE, de Groat WC, McVAry KT, et al. Tadalafil for the treatment of lower urinary tract symptoms secondary to benign prostatic hyperplasia: pathophysiology and mechanism(s) of action. Neurourol Urodyn 2011;30:292-301.

[6] Gacci M, Corona G, Vignozzi L, et al. Metabolic syndrome and benign prostatic enlargement: a systematic review and meta-analysis. BJU Int 2015;115:24-31.

[7] Vignozzi L, Rastrelli G, Corona G, et al. Benign prostatic hyperplasia: a new metabolic disease? J Endocrinol Invest 2014;37:313-22.
[8] Vignozzi L, Gacci M, Maggi M. Lower urinary tract symptoms, benign prostatic hyperplasia and metabolic syndrome. Nat Rev Urol 2016;13:108-19.

[9] Mirone V, Sessa A, Giuliano F, et al. Current benign prostatic hyperplasia treatment: impact on sexual function and management of related sexual adverse events. Int J Clin Pract 2011;65:1005-13.

[10] Gravas S, Bach T, Drake M, et al. Treatment of non-neurogenic male LUTS 2017. European Association of Urology. http://uroweb.org/ guideline/treatment-of-non-neurogenic-male-luts/.

[11] Madersbacher S, Marszalek M, Lackner J, et al. The long term outcome of medical therapy for BPH. Eur Urol 2007;51:1522-33.

[12] Giuliano F, Ückert S, Maggi M, et al. The mechanism of action of phosphodiesterase type 5 inhibitors in the treatment of lower urinary tract symptoms related to benign prostatic hyperplasia. Eur Urol 2013;63:506-16.

[13] Morelli A, Sarchielli E, Comeglio P, et al. Phosphodiesterase type 5 expression in human and rat lower urinary tract tissues and the effect of tadalafil on prostate gland oxygenation in spontaneously hypertensive rats. J Sex Med 2011;8:2746-60.

[14] Vignozzi L, Gacci M, Cellai I, et al. PDE5 inhibitors blunt inflammation in human BPH: a potential mechanism of action for PDE5 inhibitors in LUTS. Prostate 2013;73:1391-402.

[15] Morelli A, Comeglio P, Filippi S, et al. Mechanism of action of phosphodiesterase type 5 inhibition in metabolic syndrome-associated prostate alterations: an experimental study in the rabbit. Prostate 2013;73:428-41.

[16] Gacci M, Corona G, Salvi M, et al. A systematic review and metaanalysis on the use of phosphodiesterase 5 inhibitors alone or in combination with alpha-blockers for lower urinary tract symptoms due to benign prostatic hyperplasia. Eur Urol 2012;61:994-1003.

[17] Serati M, Andersson KE, Dmochowski R, et al. Systematic review of combination drug therapy for non-neurogenic lower urinary tract symptoms. Eur Urol 2019;75:129-68.

[18] Barry MJ, Fowler J, O'Leary MP, et al. The American Urological Association symptom index for benign prostatic hyperplasia. J Urol 1992;148:1549-57.

[19] Rosen RC, Riley A, Wagner G, et al. The International Index of Erectile Function (IIEF): a multidimensional scale for assessment of erectile dysfunction. Urology 1997;49:822-30.

[20] Sebastianelli A, Spatafora P, Frizzi J, et al. Tadalafil $5 \mathrm{mg}$ alone or in combination with tamsulosin $0.4 \mathrm{mg}$ for the management of men with lower urinary tract symptoms and erectile dysfunction: results of a prospective observational trial. J Clin Med 2019;8:E1126.

[21] Gacci M, Andersson KE, Chapple C, et al. Latest evidence on the use of phosphodiesterase type 5 inhibitors for the treatment of lower urinary tract symptoms secondary to benign prostatic hyperplasia. Eur Urol 2016;70:124-33.

[22] Matsukawa Y, Takai S, Majima T, et al. Objective impacts of tadalafil on storage and voiding function in male patients with benign prostatic hyperplasia: 1-year outcomes from a prospective urodynamic study. World J Urol 2018;37:867-72.

[23] Oelke M, Giuliano F, Mirone V, et al. Monotherapy with tadalafil or tamsulosin similarly improved lower urinary tract symptoms suggestive of benign prostatic hyperplasia in an international, randomised, parallel, placebo-controlled clinical trial. Eur Urol 2012;61:917-25.

[24] Oger S, Behr-Roussel D, Gorny D, et al. Combination of doxazosin and sildenafil exerts an additive relaxing effect compared with each compound alone on human cavernosal and prostatic tissue. J Sex Med 2009;6:836-47.

[25] Dong Y, Hao L, Shi Z. Efficacy and safety of tadalafil monotherapy for lower urinary tract symptoms secondary to benign prostatic hyperplasia: a meta-analysis. Urol Int 2013;91:10-8. 
[26] Urakami S, Ogawa K, Oka S, et al. Effect of tadalafil add-on therapy in patients with persistant storage symptoms refractory to $\alpha 1$-adrenoceptor antagonist monotherapy for benign prostatic hyperplasia: a randomized pilot trial comparing tadalafil and solifenacin. Low Urin Tract Symptoms 2019;11:109-14.

[27] Laydner HK, Oliveita P, Oliveira CP, et al. Phosphodiesterase 5 inhibitors for lower urinary tract symptoms secondary to benign prostatic hyperplasia: a systematic review. BJU Int 2011;107:1104-9.
[28] Singh DV, Mete UK, Mandal AK, et al. A comparative randomized prospective study to evaluate efficacy and safety of combination of tamsulosin and tadalafil vs. tamsulosin or tadalafil alone in patients with lower urinary tract symptoms due to benign prostatic hyperplasia. J Sex Med 2014;11:187-96.

[29] Barkin J, Guimarães M, Jacobi G, et al. Alpha-blocker therapy can be withdrawn in the majority of men following initial combination therapy with the dual 5alpha-reductase inhibitor dutasteride. Eur Urol 2003;44:461-6.

Please cite this article in press as: Sebastianelli A, et al. Which Drug to Discontinue 3 Months After Combination Therapy of Tadalafil plus Tamsulosin for Men with Lower Urinary Tract Symptom and Erectile Dysfunction? Results of a Prospective Observational Trial. Eur Urol Focus (2019), https://doi.org/10.1016/j.euf.2019.09.015 